\title{
Quantitative Analyse der Korallenbesiedlung eines Vorriffareals bei Aqaba (Rotes Meer)*
}

\author{
H. Mergner \& H. Schuhmacher \\ Ruhr-Universität Bochum, Lehrstuhl für Spezielle Zoologie; \\ D-4630 Bochum, Bundesrepublik Deutschland
}

\begin{abstract}
Quantitative analysis of the coral community of a fore reef area near Aqaba (Red Sea). Previous descriptions of the ecology and zonation of Aqaba reefs (Mergner \& Schuhmacher, 1974) are supplemented by this quantitative study of a test quadrat $(5 \times 5 \mathrm{~m}$ in size), randomly chosen in some $10 \mathrm{~m}$ depth in the middle fore reef of a coastal fringing reef. Of the total surface of $25 \mathrm{~m}^{2}$ Cnidaria represent $42.31 \%$, sponges $0.17 \%$, calcareous algae $0.20 \%$, dead coral rock and pebble $30.27 \%$ and sand and coral debris $26.15 \%$. The cnidarian cover is roughly equally contributed by $50.86 \%$ Scleractinia and $48.61 \%$ Alcyonaria, mainly Xeniidae $(35.81 \%)$. For each species the percentage of the total cover (measured as vertical projection), colony number, average and maximal colony size are given. A total number of 104 cnidarian species was recorded, among which the 78 scleractinian species represent 34 of the 55 coral genera known from the Red Sea. The well balanced regime of moderate light and current conditions which are tolerated both by shallow and deep water species may account for the high species number. Disturbances such as occasional sedimentation, grazing of sea urchins (Diadema setosum) and overgrowth of stony corals by xeniids result in continuous fluctuations of the coral community, in small colony size and in high colony number. Abiotic factors and biotic interactions maintain a diversity $(\mathrm{H}=3.67)$ which ranks among the greatest ever found in reef communities. The data obtained from the fore reef square are compared with those of a similar test square in the lagoon of the same reef and with results from transect zonations on the opposite coast of the Gulf of Aqaba. These comparisons indicate that the fore reef harbours the richest coral fauna in the reef. The inventory of coral species at the northern end of the Gulf of Aqaba, one of the northernmost outposts of the coral reef belt, is only little reduced when compared with that of the central Red Sea $a_{i}$ this great species diversity is in contrast to the worldwide decrease of species number towards the periphery of the reef belt.
\end{abstract}

\section{EINLEITUNG}

Artenspektrum und Artenvielfalt von Korallen und anderen sessilen Siedlern im Riff haben ökologischen und tiergeographischen Aussagewert: So charakterisieren Vergesellschaftungen, die für bestimmte Riffzonen typisch sind, und ihre zeitliche und örtliche Abfolge auch bestimmte Regime abiotischer Faktoren. In Verfolg dieser Fragestellung werden seit dem Jahre 1972 bei Aqaba am Roten Meer küstennahe Riffe untersucht. Diese Riffe stellen - als geographische Besonderheit - die nördlichsten Riffe im Roten Meer und im Indischen Ozean dar, und zugleich - nach den Riffen der Ryukyu-Inseln (Pazifik) und Bermudas (Atlantik) - die nördlichsten Riffvorkommen überhaupt.

\footnotetext{
- Mit Unterstützung der Deutschen Forschungsgemeinschaft
} 
Aufbauend auf der morphologisch-ökologischen Untersuchung zweier ganzer Riffe und der Zonierung ihrer Besiedlung (Mergner \& Schuhmacher, 1974) hatten anschlieBende Detailstudien die quantitative Erfassung produktionsbiologischer und synökologischer Zusammenhänge in genau umschriebenen Ausschnitten dieser Riffe zum Ziel (Mergner \& Svoboda, 1977; Svoboda, 1978; Mergner, 1979, 1980; Mergner \& Mastaller, 1980). Darüber hinaus liegen aus diesen Riffen Darstellungen der Mollusken- und Echinodermenfauna (Mastaller, 1979) vor, bzw. stehen hinsichtlich der Poriferen vor dem Abschluß (Nobbe, in Vorbereitung).

Schon die erste Analyse (Mergner $\&$ Schuhmacher, 1974) hatte für das Vorriff -im Vergleich mit den anderen Riffabschnitten - die größte Artenzahl und -dichte der Korallen ergeben und daher diesen Riffbereich für die Einrichtung von Testarealen $5 \times$ $5 \mathrm{~m}$ empfohlen. Die Analyse der Besiedlung durch sessiles Benthos eines dieser Areale, genannt "U-7", ist Gegenstand dieser Arbeit. Die Ergebnisse einer entsprechenden Untersuchung an einem Testareal aus der Rifflagune (L-1) sind bereits publiziert (Mergner, 1979).

\section{UNTERSUCHUNGSGEBIET UND METHODIK}

Über die allgemeinen Naturverhältnisse des Golfes von Aqaba sowie die speziellen Beobachtungen und Meßwerte im engeren Untersuchungsbereich während des Winterhalbjahres 1975/76 wurde bereits ausführlich berichtet (Mergner \& Schuhmacher, 1974; Mergner, 1979). Daher sollen an dieser Stelle nur ergänzende Angaben gemacht werden:

Das $5 \times 5 \mathrm{~m}$ große Testareal U-7 liegt im Ufersaumriff unmittelbar südlich der sog. Universitätsbucht nahe der neuen Marine Science Station Aqaba, 145-150 m von der mittleren Hochwasserlinie entfernt bei 10 bis 11,5 m Tiefe (Abb. 1). Es muß daher dem mittleren Vorriff (mit Tiefen von etwa 9 bis $23 \mathrm{~m}$ ) zugerechnet werden, dessen Boden sich im Bereich des Testareals mit etwa $20 \%$ Gefälle gegen die tiefe Mittelrinne des Golfes neigt. Es besteht teils aus plattigen Korallenfelsstrukturen mit sekundär angesiedelten, meist kleineren Korallenstöckchen, teils aus eingesenkten Sandmulden oder eingeschwemmten Korallenschuttarealen und teils aus bis zu 0,8 $\mathrm{m}$ hohen Korallenfelsaufbauten mit oft ausgedehnten Korallenkolonien (Abb. 2). Mit Ausnahme einiger Halimeda tuna-Thalli kommen im Testareal keine Makroalgen vor. Dagegen überziehen den Korallenfels zahlreiche kleine Krusten kalzifizierender Rotalgen (wahrscheinlich Lithothamnion und Porolithon) oder Rasen feinfädiger Braunalgen.

Das Testareal U-7 gehört dem Bereich der Strömungszone an mit einer in $10 \mathrm{~m}$ Tiefe vorwiegend nordwärts setzenden Rifflängsströmung, mit seitlicher Wasserversetzung ohne Pendelbewegung und mit meist mäßigen Strömungsgeschwindigkeiten, die in der Regel $5 \mathrm{~cm} \mathrm{~s}^{-1}$ nicht überschreiten. Lediglich bei länger anhaltenden stürmischen Südwinden können höhere Geschwindigkeiten und dann auch Pendelbewegungen auftreten, ohne aber in dieser Tiefe unmittelbar zerstörende Wirkungen auf die Korallenbesiedlung auszuüben. Allerdings vermag der damit verbundene stärkere Sedimenttransport kleinere Korallenkolonien durch Uberdecken mit Sand zu schädigen, wofür zahlreiche tote Korallenstückchen im Untersuchungsgebiet sprechen. Nobbe (in Vorbereitung) hat in der gleichen Riffregion südlich Aqaba entsprechende Schäden auch an Poriferen festgestellt und daraufhin die Sedimentationsrate gemessen. 
Dank der beständigen, mäßigen Wasserbewegung ist auch die Schwankungsbreite von Temperatur, Salinität und Sauerstoffgehalt gering. So betragen die Tag-NachtUnterschiede der Temperatur im mittleren Vorriff bei $10 \mathrm{~m}$ Tiefe kaum mehr als $\pm 0,3^{\circ} \mathrm{C}$ und die der Salinität weniger als $0,1 \%$. Der $\mathrm{O}_{2}$-Gehalt schwankt dabei nur um $0,5 \mathrm{mgl}^{-1}$; er lag im Februar 1976 zwischen 8,2 und 8,7 $\mathrm{mgl}^{-1}$ (Mergner \& Svoboda, 1977). Im gleichen Zeitraum betrug die Beleuchtungsintensität im $10 \mathrm{~m}$ tiefen mittleren Vorriff um die Mittagszeit $18 \mathrm{mWcm}^{-2}$ (Svoboda, 1978). Sie war damit ausreichend zur Produktion eines $\mathrm{O}_{2}$-Überschusses und für eine P/R-Rate von mehr als 1,0 bei allen 12 daraufhin überprüften zooxanthellenhaltigen Korallenarten aus dem Riffgebiet südlich Aqaba.
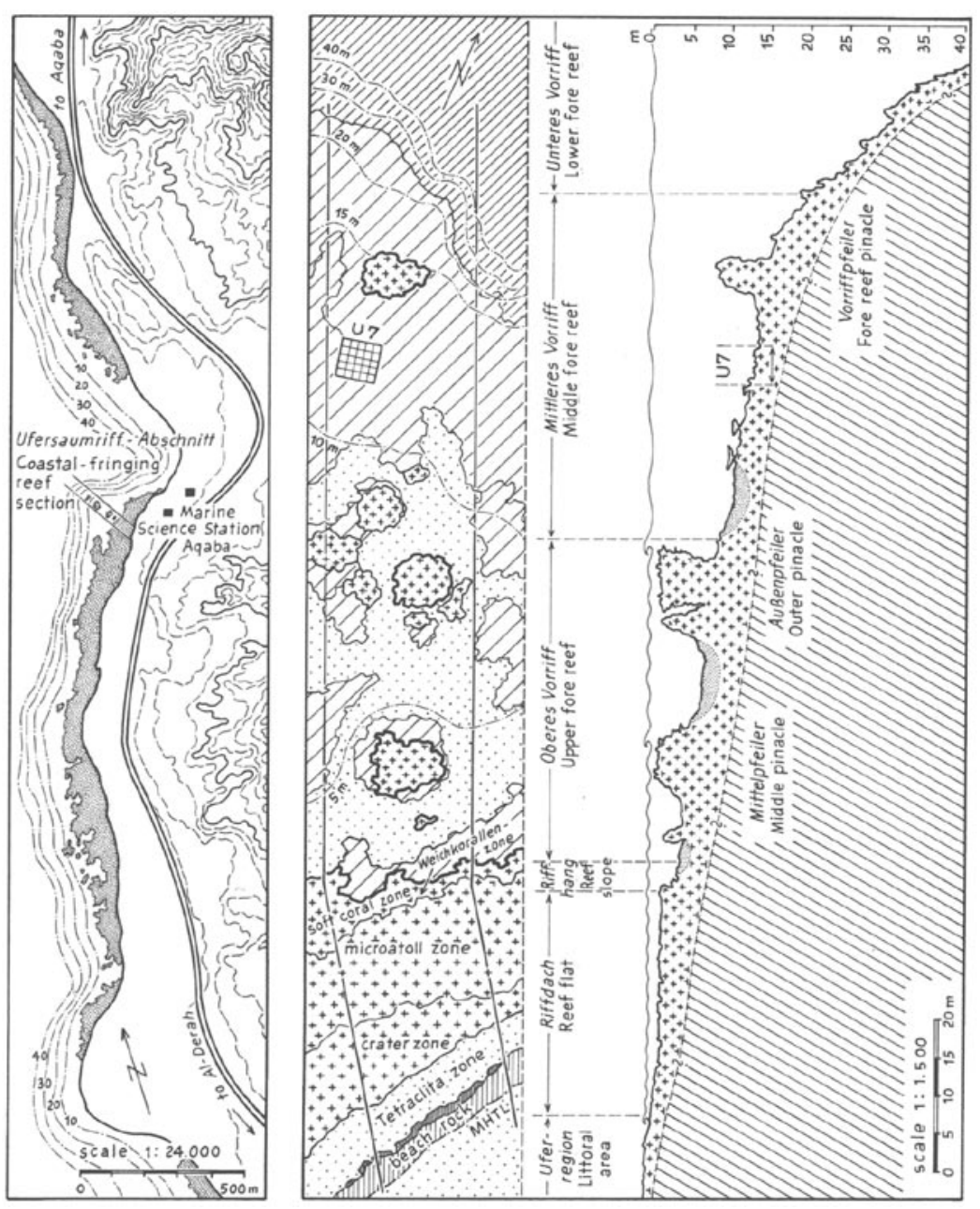

Abb. 1. Untersuchungsregion bei Aqaba mit dem "Universitätsgelände" unmittelbar südlich der Marine Science Station (links). Untersuchter Abschnitt des Ufersaumriffes mit Lage des Testareals U-7 in der Aufsicht (Mitte) und im Profil (rechts). (Nach Mergner \& Schuhmacher, 1974, und Mergner \& Svoboda, 1977, verändert) 
Angesichts der oft bedeutenden Ausdehnung eines Korallenriffes können quantitative Erhebungen nur an repräsentativen Stichproben gemacht werden, die sich grundsätzlich entweder mit der Transekt- oder mit der Quadratmethode gewinnen lassen. Während die Transektmethode schneller eine Abfolge verschiedener Vergesellschaftungen verdeutlichen kann, eignet sich die Quadratmethode besonders, um eine bestimmte Zone in ihrer Artenzusammensetzung wiederzugeben (vgl. auch Goodwin et al., 1976). Doch ist - von Größe und Artenzahl der Korallenkolonien abhängig - eine Mindestquadratgröße notwendig, um repräsentativ zu sein; sie läßt sich im Einzelfalle nur empirisch bestimmen. Daher variieren die Flächenmaße der von verschiedenen Autoren bisher festgelegten Testquadrate erheblich: 0,25 $\mathrm{m}^{2}$ bei Neudecker (1976), 0,5 $\mathrm{m}^{2}$ bei Ott \& Auclair (1977), $1 \mathrm{~m}^{2}$ bei Maragos (1974), Morton (1974) und Dinesen (1977), $5 \mathrm{~m}^{2}$ bei Salvat (1972), 9,25 $\mathrm{m}^{2}$ bei Davies et al. (1971) und $25 \mathrm{~m}^{2}$ bei Mergner et al. (1977, 1979,1980 ).

Die von uns gewählte Dimension von $5 \times 5 \mathrm{~m}$ war schon insofern vorgegeben, als dieses Quadrat U-7 mit anderen Testquadraten im Riff (z. B. L-1) vergleichbar sein sollte. Bei letzteren war aber ein ausreichendes Areal notwendig, um zugleich auch vagile Formen (wie etwa Gastropoden und Echinodermen) über einen gewissen Zeitraum hinweg quantitativ erfassen zu können. Inzwischen wurden auch entsprechende Erhebungen an sechs gleichgroßen Quadraten in küstenfernen Riffen bei Port Sudan durchgeführt. Nach Auswertung dieser Ergebnisse wird ein Vergleich beider Regionen des Roten Meeres möglich sein.

Ein quantitatives Erfassen von Flächenbedeckungen im Riff ist wegen dessen sehr unregelmäßiger Oberflächenskulpturierung nur unzulänglich möglich. Näherungsmethoden zur Flächenerfassung dreidimensionaler Hartbodenstrukturen (Dahl, 1973; Pichon, 1978) erscheinen daher nur für bestimmte, geometrisch regelmäßige Wuchsformen sinnvoll, die sich jedoch in der vielgestaltigen Rifflandschaft nur ausnahmsweise finden. Sofern allerdings die Frage nach der Artendiversität und -zusammensetzung im Vordergrund steht, ist folgendes Verfahren anwendbar, wonach lediglich die Projektion der dreidimensionalen Strukturen in ihre Grundebene berücksichtigt wird. Bei dieser vielfach gebrauchten Methode wird in Kauf genommen, daß die resultierende Flächenmaßzahl kleiner als die tatsächliche Oberfläche ist. Die Bedeckungsrate läßt sich - wie auch von Bohnsack (1979) bestätigt - photographisch hinreichend genau und vor allem rasch erfassen, was gerade hinsichtlich der begrenzten Tauchzeiten von Bedeutung ist.

Zur genauen Lokalisierung aller Strukturen und Flächenberechnung der kolonial wachsenden Arten wurde das Testareal mit einem $5 \times 5 \mathrm{~m}$-Netz aus Perlonleinen von 1 $\mathrm{m}$ Maschenweite überspannt und für die Flächenprojektion jedes der 25 Teilquadrate von $1 \mathrm{~m}^{2}$ Fläche zentrisch aus gleichem Abstand $(2 \mathrm{~m})$ mit einer Unterwasserkamera (Nikonos "Calypso", 28 mm Brennweite) und Elektronenblitz photographiert (Abb. 5a). Die photographische Erfassung des Testareals und eine erste Aufsammlung von Belegexemplaren fanden im Februar 1976 statt; im September 1977 wurden von M. Mastaller weitere Belegstücke entnommen und dabei auch die inzwischen erfolgten Veränderungen im Besiedlungsbild registriert.

Die Einzelquadrate wurden dann zeichnerisch zusammengefaßt und ihre Korallenkolonien nach Photographien und Belegstücken determiniert. Durch Kartieren der Einzelkolonien ergab sich schließlich ein fast lückenloses Bild von der Besiedlungsstruktur der Cnidarier im Testareal. Um den relativen Anteil der jeweiligen Bodensub- 
strate und Sedentarierarten an der gesamten Arealfläche zu ermitteln, wurden in der Kartenzeichnung die Einzelflächen und Einzelkolonien entlang ihrer Umrisse ausgeschnitten und ihr Gewicht zu dem der Gesamtfläche in Beziehung gesetzt.

Zur Berechnung der Artendiversität und zahlenmäßigen Abundanz wurde der Shannon-Index (vgl. Pielou, 1969) verwendet:

$$
H=-\sum_{i=1}^{\mathrm{N}} p_{i} \cdot \ln p_{i}
$$

Dabei bedeuten $\mathrm{N}$ die Artenzahl und $p_{i}$ die gemessene Individuenrate.

\section{ERGEBNISSE}

Die zeichnerische Auswertung der gesamten Photoserie gibt zunächst einen Eindruck von der strukturellen Gliederung des Untersuchungsareals (Abb. 2). Die Korallenaufbauten erheben sich in diesem Bereich nicht mehr als 0,6 $\mathrm{m}$ über den sandbedeckten Boden. Auffällig hochragende oder weit vorkragende Skelettstrukturen, wie sie für den Riffhang charakteristisch sind, fehlen hier. Das Bodenprofil ist vielmehr durch niedrige, hauptsächlich buckelig-krustige Erhebungen und eingesprengte Sandflächen mit eingeschwemmtem Korallenschutt gekennzeichnet. Im Gegensatz zu den steiler abfallenden Abschnitten des unteren Vorriffes steht im mittleren Vorriff kein gewachsener Fels sichtbar an.

Die artenmäßige Zusammensetzung des Cnidarierbestandes im untersuchten Ausschnitt U-7 wird auf Abb. 2 und 3 bei senkrechter Projektion lagerichtig und flächentreu wiedergegeben. Diese Karte ist Grundlage für verschiedene quantitative Auswertungen (Tab. 1 und 2): Danach sind fast $43 \%$ der Bodenfläche von lebenden sessilen Organismen bedeckt, und zwar fast ausschließlich von Cnidaria (42,31\% der Gesamtfläche). Dagegen sind Kalkrotalgen $(0,20 \%)$, Porifera $(0,17 \%)$, Polychaeta sedentaria, Bryozoa und Tunicata, die in manchen Riffgebieten neben den Cnidaria noch eine gewisse Rolle bei der Zusammensetzung des sessilen Benthos spielen, in nur geringem Maße vertreten - und wenn, dann unter Korallenkolonien verborgen. Eine Analyse der CnidariaPopulation zeigt, daß unter dieser die Scleractinia mit $21,49 \%$ und die Alcyonaria mit $20,62 \%$ der Gesamtfläche, bzw. mit 50,86\% und 48,61 \% an der Cnidaria-Bedeckung, nahezu gleichgroße_Areale besiedeln. Dabei stellen die Xeniidae mit $15,21 \%$ an der Gesamtfläche $(35,81 \%$ an der Cnidaria-Bedeckung) den überragenden Anteil. Alle anderen Cnidaria-Gruppen (Hydroidea 0,09\% der Gesamtfläche, Rhizostomeae 0,03\%, Stolonifera $0,02 \%$ und Zoantharia $0,03 \%$ ) sind mit zusammen $0,17 \%$ ohne Bedeutung. $30,27 \%$ Korallenfels und Gerölle sowie $26,15 \%$ Sand und Korallengrus bilden das leblose Substrat der Gesamtfläche.

Von Interesse sind auch Kolonienzahl, Größenverhältnisse und Zugehörigkeit der Scleractinia-Kolonien zu den verschiedenen Wuchstypen (Tab, 2): So beträgt der Anteil der Zweigkorallen (Pocillopora, Seriatopora, Stylophora und Acropora) mit $12,37 \%$ (220 Kolonien) aller lebenden Cnidarier einerseits und der der krustenbildenden Korallen (Psammocora, Montipora, Pavona, Echinophyllia, Mycedium, Turbinaria) mit 14,18\% (323 Kolonien) anderseits zusammen nur etwa so viel wie derjenige der massig wach- 
Tab. 1. Quantitative tabellarische Arlalyse der Cnidariergemeinschaft aus Testareal U-7

\begin{tabular}{|c|c|c|c|c|c|c|c|}
\hline Nr. & Order, Genus, Species & 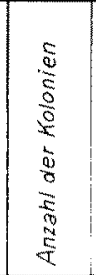 & 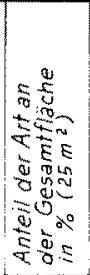 & 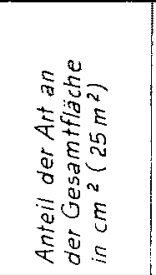 & 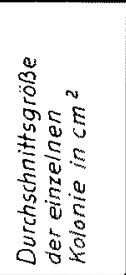 & 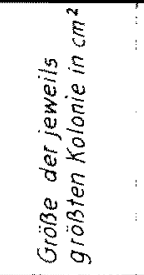 & 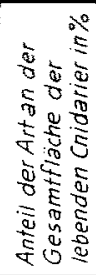 \\
\hline \multirow{5}{*}{1} & Hydroidea & 16 & 0.09 & 241.94 & & & 0.23 \\
\hline & Millepora exaesa & 16 & 0.09 & 241.94 & 15.12 & 43.38 & 0.23 \\
\hline & Rhizostomeae & 2 & 0.03 & 83.18 & & & 0.08 \\
\hline & Cassiopea andromeda & 2 & 0.03 & 83.18 & 41.59 & 58.13 & 0.08 \\
\hline & Stolonifera & 2 & 0.02 & 43.38 & & & 0.04 \\
\hline \multirow[t]{2}{*}{2} & Tubipora musica & 2 & 0.02 & 43.38 & 21.69 & 22.80 & 0.04 \\
\hline & Alcyonaria & 749 & 20.62 & 51631.28 & & & 48.61 \\
\hline 3 & Cladiella pachyclados & 12 & 0.14 & 393.89 & 28.32 & 54.11 & 0.32 \\
\hline 4 & Lobophytum sp. & 1 & 0.01 & 25.04 & 25.04 & 25.04 & 0.02 \\
\hline 5 & Parerythropodium fulvum & 92 & 1.02 & 255769 & 27.80 & 120.75 & 2.41 \\
\hline 6 & Sarcophyton ehrenbergi & 28 & 3.26 & 8161.89 & 291.49 & 1451.69 & 7.69 \\
\hline 7 & Sinularia leptoclados & 2 & 0.04 & 109.12 & 54.56 & 90.78 & 0.10 \\
\hline $8:$ & Sinularia polydactyla + sp. & 2 & 0.03 & 63.95 & 31.97 & 44.72 & 0.06 \\
\hline 9 & Dendronephthya sp. & 3 & 0.02 & 47.40 & 15.80 & 34.43 & 0.05 \\
\hline 10 & Nephthea albida & 1 & 0.03 & 80.50 & 80.50 & 80.50 & 0.08 \\
\hline 11 & Lithophyton arboreum & 3 & 0.80 & 1997.76 & 665.92 & 80992 & 1.88 \\
\hline 12 & Paralemnalia thyrsoides & 4 & 0.06 & 161.44 & 40.36 & 58.13 & 0.15 \\
\hline 13 & Heteroxenia fuscescens. & 29 & 0.93 & 2333.18 & 80.45 & 315.74 & 2.20 \\
\hline 14 & Xenia macrospiculata & 232 & 5.70 & 14239.70 & 61.37 & 363.59 & 13.41 \\
\hline 15 & Xenia membranacea & 8 & 0.13 & 330.94 & 41.36 & 239.26 & 0.31 \\
\hline 16 & Xenia obscuronata & 61 & 2.59 & 6463.77 & 105.96 & 516.99 & 6.09 \\
\hline 17 & Xenia spec. & 69 & 0.73 & 1832.73 & 26.56 & 98.38 & 1.73 \\
\hline \multirow[t]{2}{*}{18} & Xenia umbellata & 202 & 5.13 & 12832.28 & 63.52 & 1024.15 & 12.11 \\
\hline & Scleractinia & 1274 & 21.49 & 53905.19 & & & 50.86 \\
\hline 19 & Psammocora superficialis & 4 & 0.07 & 173.07 & 43.26 & 90.33 & 0.16 \\
\hline 20 & Pocillopora damicornis & 3 & 0.05 & 127.90 & 31.97 & 68.42 & 0.12 \\
\hline 21 & Pocillopora danae & 2 & 0.05 & 124.32 & 62.16 & 56.79 & 0.12 \\
\hline 22 & Seriatopora angulata & 5 & 0.13 & 318.87 & 63.77 & 176.65 & 0.30 \\
\hline 23 & Seriatopora caliendrum & 1 & 0.01 & 17.88 & 17.88 & 17.88 & 0.02 \\
\hline 24 & Seriatopora sp. & 2 & 0.02 & 47.85 & 23.92 & 33.54 & 0.05 \\
\hline 25 & Stylophora pistillata & 94 & 1.61 & 4031.30 & 42.88 & 169.94 & 3.80 \\
\hline 26 & Acropora clavigera & 13 & 0.14 & 357.33 & 27.48 & 122.98 & 0.34 \\
\hline 27 & Acropora eurystoma & 1 & 0.23 & 569.31 & 569.31 & 569,31 & 0.54 \\
\hline 28 & Acropora forskali & 1 & 0.01 & 30.41 & 30.41 & 30.41 & 0.03 \\
\hline 29 & Acropora hemprichi & 6 & 0.09 & 221.37 & 36.89 & 71.55 & 0.21 \\
\hline 30 & Acropora humilis & 4. & 0.09 & 229.42 & 57.35 & 137.74 & 0.22 \\
\hline 31 & Acropora scandens & 2 & 0.06 & 150.26 & 75.13 & 76.92 & 0.14 \\
\hline 32 & Acropora squarrosa & 44 & 1.79 & 4474.50 & 101.69 & 614.93 & 4.22 \\
\hline
\end{tabular}


Tab. 1 (Fortsetzung)

\begin{tabular}{|c|c|c|c|c|c|c|c|}
\hline Nr. & Order, Genus, Species & 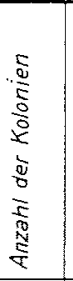 & 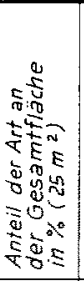 & 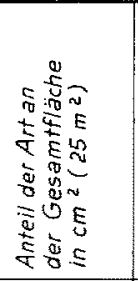 & 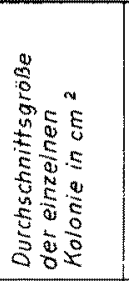 & 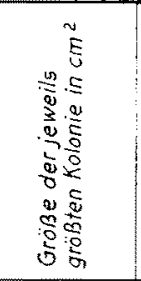 & 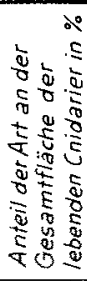 \\
\hline 33 & Acropora variabilis & 32 & 0.92 & 2287.56 & 71.48 & 345.10 & 2.15 \\
\hline 34 & Acropora sp. & 10 & 0.06 & 145.34 & 14.53 & 30.41 & 0.14 \\
\hline 35 & Astreopora myriophthalma & 17 & 0.15 & 369.40 & 21.72 & 80.50 & 0.35 \\
\hline 36 & Montipora danae & 11 & 0.10 & 256.26 & 23.29 & 83.63 & 0.24 \\
\hline 37 & Montipora effusa & 2 & 0.11 & 279.06 & +39.53 & 142.21 & 0.26 \\
\hline 38 & Montipora eilatensis & 3 & 0.04 & 99.73 & 33.24 & 47.85 & 0.09 \\
\hline 39 & Montipora meandrina & 62 & 1.07 & 2686.93 & 43.33 & 275.93 & 2.53 \\
\hline 40 & Montipora monasteriata & 23 & 0.35 & 873.43 & 37.97 & 92.57 & 0.82 \\
\hline 41 & Montipora stilosa & 61 & 2.10 & 5257.60 & 86.19 & 322.00 & 4.95 \\
\hline 42 & Montipora venosa & 4 & 0.10 & 245.52 & 61.38 & 68.42 & 0.23 \\
\hline 43 & Montipora sp. & 84 & 0.94 & 2425.75 & 28.87 & 181.57 & 2.28 \\
\hline 44 & Gardineroseris ponderosa & 4 & 0.11 & 281.75 & 70.43 & 123.43 & 0.27 \\
\hline 45 & Leptoseris sp. & 8 & 0.10 & 237.92 & 29.74 & 68.42 & 0.22 \\
\hline 46 & Pachyseris speciosa & 1 & 0.01 & 31.75 & 31.75 & 31.75 & 0.03 \\
\hline 47 & Pavona danae & 5 & 0.06 & 148.03 & 29.60 & 63.95 & 0.14 \\
\hline 48 & Pavona decussata & 4 & 0.04 & 110.46 & 2761 & 49.64 & 0.10 \\
\hline 49 & Pavona varians & 41 & 0.64 & 160554 & 39.15 & 231.21 & 1.51 \\
\hline 50 & Coscinarea monile & 4 & 0.14 & 347.49 & 86.87 & 168.60 & 0.33 \\
\hline 51 & Cycloseris doederleini & 2 & 0.02 & 61.71 & 30.85 & 38.90 & 0.06 \\
\hline 52 & Cycloseris sp. & 1 & 0.01 & 2325 & 23.23 & 23.25 & .02 \\
\hline 53 & Fungia fungites & 7 & 0.08 & 188.72 & 26.96 & 55.00 & 0.18 \\
\hline 54 & Fungia granulosa & 6 & 0.05 & 130.59 & 26.11 & 40.25 & 0.12 \\
\hline 55 & Alveopora daedalea & 24 & 0.35 & 872.09 & 36.33 & 139.08 & 0.82 \\
\hline 56 & Goniopora planulata & 34 & 2.03 & 5080.05 & 149.41 & 1301.43 & 4.78 \\
\hline 57 & Goniopora sp. & 12 & 0.24 & 587.66 & 48.97 & 177.54 & 0.55 \\
\hline 58 & Porites (Synarea) convexa & 4 & 0.11 & 281.75 & 70.43 & 111.80 & 0.27 \\
\hline 59 & Porites lutea & 11 & 0.23 & 571.10 & 51.91 & 152.95 & 0.54 \\
\hline 60 & Porites sp. & 23 & 0.15 & 385.95 & 16.78 & 52.32 & 36 \\
\hline 61 & Cautastrea tumida & 2 & 0.31 & 770.57 & 385.29 & 35465 & 0.73 \\
\hline 62 & Cyphastrea chalcidicum & 16 & 0.17 & 429.33 & 26.83 & 96.60 & 0.40 \\
\hline 63 & Cyphastrea microphthalma & 27 & 0.28 & 690.51 & 25.57 & 55.00 & 0.65 \\
\hline 64 & Cyphastrea serallia & 2 & 0.02 & 49.19 & 24.59 & 35.77 & 0.05 \\
\hline 65 & Cyphastrea sp. & 1 & 0.01 & 17.88 & 17.88 & 17.88 & 0.02 \\
\hline 66 & Echinopora gemmacea & 24 & 0.32 & 812.16 & 33.84 & 138.64 & 0.76 \\
\hline 67 & Echinopora gemmacea fruticulosa & 8 & 0.11 & 268.33 & 33.54 & 137.29 & 0.25 \\
\hline 68 & Echinopora lamellosa & 1 & 0.02 & 50.08 & 50.08 & 50.08 & 0.05 \\
\hline 69 & Favia amicorum & 5 & 0.07 & 162.79 & 32.55 & 4293 & 0.15 \\
\hline 70 & Favia favus & 25 & 0.24 & 592.57 & 23.70 & 63.95 & 0.56 \\
\hline
\end{tabular}


Tab. 1 (Fortsetzung)

\begin{tabular}{|c|c|c|c|c|c|c|c|}
\hline Nr. & Order, Genus, Species & 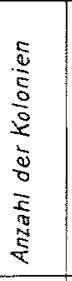 & 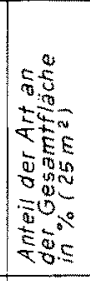 & 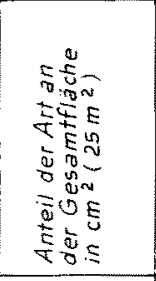 & 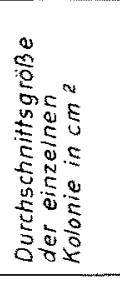 & 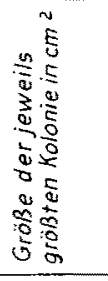 & 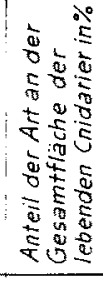 \\
\hline 71 & Favia laxa & 7 & 0.07 & 330.50 & 47.21 & 45.16 & 0.31 \\
\hline 72 & Favia pallida & 3 & 0.03 & 77.81 & 25.93 & 40.25 & 0.07 \\
\hline 73 & Favia stelligera & 9 & 0.20 & 505.36 & 56.15 & 125.67 & 0.48 \\
\hline 74 & Favia sp. & 16 & 0.09 & 234.79 & 14.67 & 49.19 & 0.22 \\
\hline 75 & Favites abdita & 7 & 0.09 & 232.55 & 33.22 & 49.19 & 0.22 \\
\hline 76 & Favites peresi & 65 & 0.50 & 1239.26 & 19.06 & 64.40 & 1.17 \\
\hline 77 & Favites sp. & 25 & 0.17 & 436.94 & 17.47 & 65.74 & 0.41 \\
\hline 78 & Goniastrea edwardsi & 1 & 0.01 & 12.52 & 12.52 & 12.52 & 0.01 \\
\hline 79 & Goniastrea pectinata & 162 & 1.68 & 4195.43 & 25.89 & 308.13 & 3.95 \\
\hline 80 & Goniastrea retiformis & 27 & 0.35 & 880.58 & 32.61 & 111.80 & 0.83 \\
\hline 81 & Goniastrea sp. & 7 & 0.13 & 328.26 & 46.89 & 90.78 & 0.31 \\
\hline 82 & Hydnophora exaesa & 4 & 0.05 & 112.70 & 28.17 & 51.87 & 0.11 \\
\hline 83 & Leptastrea bottae & 58 & 0.63 & 1573.34 & 393.33 & 178.89 & 1.48 \\
\hline 84 & Leptastrea purpurea & 3 & 0.01 & 33.09 & 11.03 & 13.86 & 0.03 \\
\hline 85 & Leptastrea transversa & 11 & 0.10 & 242.39 & 22.03 & 46.51 & 0.23 \\
\hline 86 & Montastrea forskaelana & 15 & 0.26 & 639.53 & 42.63 & 206.61 & 0.60 \\
\hline 87 & natygyra lamellina & 14 & 0.19 & 487.47 & 34.81 & 95.25 & 0.46 \\
\hline 88 & Galaxea fascicularis & 6 & 0.06 & 160.10 & 26.68 & 37.11 & 0.15 \\
\hline 89 & Merulina ampliała & 1 & 0.01 & 24.15 & 24.15 & 24.15 & 0.02 \\
\hline 90 & Acanthastrea echinata & 5 & 0.02 & 40.69 & 8.13 & 12.52 & 0.08 \\
\hline 99 & Lobophyllia corymbosa & 2 & 0.03 & 63.50 & 31.75 & 33.09 & 0.06 \\
\hline 92 & Lobophyllia costata & 8 & 0.16 & 402.50 & 50.31 & 129.69 & 0.38 \\
\hline 93 & Lobophyllia hemprichi & 2 & 0.01 & 10.28 & 5.14 & 4.91 & 0.01 \\
\hline 94 & Echinophyllia aspera & 1 & 0.12 & 292.03 & 292.03 & 292.03 & 0.27 \\
\hline 95 & Mycedium tubifex & 18 & 0.26 & 638.19 & 35.45 & 69.76 & 0.60 \\
\hline \multirow[t]{2}{*}{96} & Turbinaria mesenterina & 4 & 0.05 & 121.64 & 30.41 & 78.26 & 0.11 \\
\hline & Zoantharia & 3 & 0.03 & 65.29 & & & 0.06 \\
\hline \multirow[t]{5}{*}{97} & Palythoa tuberculosa & 3 & 0.03 & 65.29 & 21.76 & 42.48 & 0.06 \\
\hline & Kalkalgen & 25 & 0.20 & 494.18 & 19.76 & 87.20 & \\
\hline & Schwämme & 7 & 0.17 & 429.33 & 61.33 & 211.98 & \\
\hline & Korallenfels u. Gerölle & & 30.27 & 76840.75 & & & \\
\hline & Sand und Korallengrus & & 26.15 & 65383.24 & & & \\
\hline
\end{tabular}


Tab. 2. Quantitative tabellarische Gesamtanalyse der Cnidariergemeinschaft aus Testareal U-7 mit den wichtigsten systematischen Kategorien und Wuchstypen sowie der übrigen Bodenbedeckung

\begin{tabular}{|c|c|c|c|c|}
\hline Kategorien der Bodenbedeckung & $\begin{array}{l}5 \\
\frac{5}{5} \\
0 \\
0 \\
1 \\
\frac{0}{0} \\
0 \\
0 \\
\frac{1}{0} \\
\frac{1}{N} \\
\frac{1}{2}\end{array}$ & 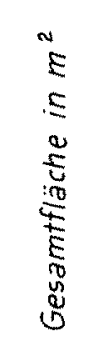 & 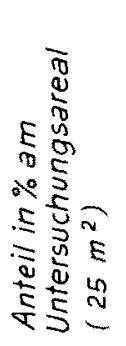 & 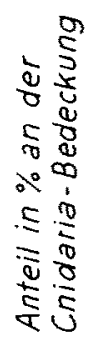 \\
\hline Anthozoa, systematische Gruppen & & & & \\
\hline Alcyonaria & 749 & 5.16 & 20.62 & 48.61 \\
\hline Xeniidae & 601 & 3.80 & 15.21 & 35.81 \\
\hline Scleractinia & 1274 & 5.39 & 21.57 & 50.86 \\
\hline Astrocoeniina & 491 & 2.58 & 10.32 & 24.29 \\
\hline Fungiina & 124 & 1.10 & 4.37 & 10.32 \\
\hline Faviina & 569 & 1.70 & 6.57 & 16.04 \\
\hline $\begin{array}{c}\text { Anthozoa, Gesamtzahl der Kolonien } \\
\% \text { Bedeckung }\end{array}$ & 2028 & 10.60 & 42.24 & 99.88 \\
\hline Korallen, Wuchstypen & & & & \\
\hline Zweigkorallen & 220 & 1.31 & 5.25 & 12.37 \\
\hline Krustige Formen (überwiegend Montipora) & 323 & 1.50 & 6.00 & 14.18 \\
\hline $\begin{array}{l}\text { Massig wachsende Kolonien (Poritidae + Faviina) } \\
\text { Andersartige Bodenbedeckung }\end{array}$ & 711 & 2.46 & 10.02 & 23.37 \\
\hline Lebende Bedeckung & 32 & 0.09 & 0.37 & - \\
\hline Kalkrotalgen (Lithothamnion + Porolithon) & 25 & 0.05 & 0.20 & - \\
\hline Porifera & 7 & 0.04 & 0.17 & - \\
\hline Leblose Substrate & - & 14.22 & 56.42 & - \\
\hline Korallenfels & - & 7.63 & 30.05 & - \\
\hline Sand & - & 6.54 & 26.15 & - \\
\hline Geröll & - & 0.05 & 0.22 & - \\
\hline Gesamtfläche & - & 24.98 & 99.07 & 99.88 \\
\hline Differenz zur Gesamtsumme & - & -0.02 & -0.93 & -0.12 \\
\hline
\end{tabular}


Tab. 3. Quantitative tabellarische Analyse der Veränderungen im Cnidarierbestand des Testareals U-7 mit prozentualen und flächenmäßigen Anteilen der zwischen Frühahr 1976 und September 1977 abgestorbenen Kolonien

\begin{tabular}{|c|c|c|c|c|c|c|}
\hline 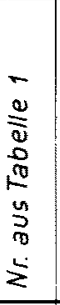 & Order, Genus, Species & 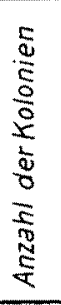 & 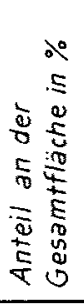 & 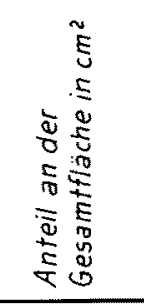 & 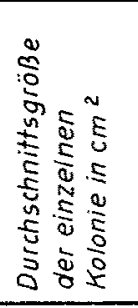 & 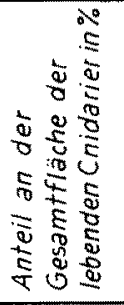 \\
\hline & Alcyonaria & 27 & 0.36 & 852.50 & 29.05 & 0.69 \\
\hline 5 & Parerythropodium fulvum & 2 & 0.02 & 45.00 & 22.50 & 0.04 \\
\hline 6 & Sarcophyton ehrenbergi & 2 & 0.02 & 45.00 & 22.50 & 0.04 \\
\hline 14 & Xenia macrospiculata & 7 & 0.11 & 267.50 & 38.21 & 0.21 \\
\hline 16 & xenia obscuronata & 5 & 0.07 & 170.00 & 34.00 & 0.14 \\
\hline 17 & xenia spec. & 6 & 0.10 & 237.50 & 39.58 & 0.19 \\
\hline 18 & Xenia umbellata & 5 & 0.04 & 87.50 & 17.50 & 0.07 \\
\hline & Scleractinia & 53 & 0.81 & 1912.50 & 39.85 & 1. 54 \\
\hline 22 & Seriatopora angulata & 1 & 0.06 & 152.50 & 152.50 & 0.12 \\
\hline 25 & Stylophora pistillata & 4 & 0.05 & 112.50 & 28.12 & 0.09 \\
\hline 32 & Acropora squarrosa & 1 & 0.03 & 77.50 & 77.50 & 0.06 \\
\hline 33 & Acropora variabilis & 1 & 0.01 & 5.00 & 5.00 & 0.01 \\
\hline 35 & Astreopora myriophthalma & 2 & 0.01 & 32.50 & 16.25 & 0.03 \\
\hline 39 & Montipora meandrina & 3 & 0.06 & 157.50 & 52.50 & 0.13 \\
\hline 41 & Montipora stilosa & 4 & 0.11 & 267.50 & 66.87 & 0.21 \\
\hline 43 & Montipora sp. & 2 & 0.01 & 20.00 & 10.00 & 0.02 \\
\hline 45 & Leptoseris sp. & 1 & 0.02 & 40.00 & 40.00 & 0.03 \\
\hline 49 & Pavona varians & 3 & 0.05 & 120.00 & 40.00 & 0.10 \\
\hline 53 & Fungia fungites & 1 & 0.01 & 17.50 & 17.50 & 0.01 \\
\hline 55 & Alveopora daedalea & 2 & 0.05 & 112.50 & 56.25 & 0.09 \\
\hline 57 & Goniopora sp. & 2 & 0.01 & 32.50 & 16.25 & 0.03 \\
\hline 58 & Porites (Synarea) convexa & 3 & 0.03 & 70.00 & 23.33 & 0.06 \\
\hline 59 & Porites lutea & 1 & 0.01 & 12.50 & 12.50 & 0.01 \\
\hline 60 & Porites sp. & 2 & 0.02 & 52.50 & 26.25 & 0.04 \\
\hline 63 & Cyphastrea microphthalma & 1 & 0.01 & 17.50 & 17.50 & 0.01 \\
\hline 66 & Echinopora gemmacea & 2 & 0.02 & 40.00 & 20.00 & 0.03 \\
\hline 70 & Favia favus & 4 & 0.04 & 87.50 & 21.87 & 0.07 \\
\hline 74 & Favia sp. & 1 & 0.04 & 95.00 & 95.00 & 0.08 \\
\hline 76 & Favites peresi & 5 & 0.05 & 125.00 & 25.00 & 0.10 \\
\hline 79 & Goniastrea pectinata & 6 & 0.08 & 202.50 & 33.75 & 0.16 \\
\hline \multirow[t]{3}{*}{83} & Leptastrea bottae & 1 & 0.03 & 62.50 & 62.50 & 0.05 \\
\hline & Alcyonaria + Scleractinia & 80 & 1.17 & 2765.00 & 34.45 & - \\
\hline & Kalkalgen & 6 & 0.03 & 62.50 & 10.41 & 0.05 \\
\hline
\end{tabular}


senden Arten (Astreopora, Gardineroseris, Leptoseris, Pachyseris, Coscinarea, Poritidae und Faviina ohne Pectiniidae) mit $23,37 \%$ (711 Kolonien). Die Einzelkorallen (Cycloseris und Fungia) stellen demgegenüber nur 0,16\% (16 Kolonien).

Insgesamt wurden 2028 Kolonien bzw. Individuen gezählt (Tab. 1). Hierbei muß aber eingeräumt werden, daß unmittelbar benachbarte Kolonien der gleichen Art - so bei Goniopora planulata - nicht immer eindeutig unterschieden werden konnten. Vor allem die aus vielen Einzelkolonien bestehenden Xenia-Rasen konnten naturgemäß nicht aufgelöst werden; hingegen werden zwei getrennte, lebende Partien einer alten, teilweise abgestorbenen Korallenkolonie als zwei Einheiten gewertet. Angesichts der hohen Anzahl von Cnidarier-Kolonien auf einer Fläche von $10,63 \mathrm{~m}^{2}$ ist die durchschnittliche Koloniegröße gering; nur bei den wenigen (ausgewachsenen) Weichkorallen Sarcophyton ehrenbergi und Lithophyton arboreum ergeben sich Abweichungen nach oben. Die meisten Steinkorallen bleiben hingegen weit unter der Koloniegröße, die von ihren Arten am Riffhang erreicht werden. Dies gilt vor allem für Hydnophora exesä, Platygyra lamellina, verschiedene Favia-Arten und Porites spp. Hingegen erreichen Acropora squarrosa, Montipora effusa und Goniopora planulata im Vorriff ihre maximale Größe. Bei genauerer Prüfung zeigt es sich allerdings oft, daß Einzelkolonien

Tab. 4. Liste der veröffentlichten (Mergner \& Schuhmacher, 1974; Mergner \& Wedler, 1977; Mergner, 1979), nicht in Tab. 1 aufgeführten Cnidaria-Arten

Hydroidea:

Dynamena cornicina*

Gymnangium eximium

Halocordyle disticha*

Millepora dichotoma

Millepora platyphylla

Solanderia secunda*

A lcyonaria:

Dendronephthya hemprichi

Sarcophyton glaucum

Sinularia gardineri

Sinularia querciformis

Xenia mayi

Gorgonaria:

Acabaria pulchra

Actiniaria:

Boloceroides mc murrichi

Cryptodendrum adhesivum

Megalactis hemprichi

Triactis producta

Ceriantharia:

Cerianthus sp.*
Scleractinia:

Acropora corymbosa

Balanophyllia gemmifera

Favia matthai

Favia speciosa

Favites chinensis

Favites complanata

Favites pentagona

Favites melicerum *

Favites virens

Fungia scutaria

Gyrosmilia interrupta

Herpolitha limax

Hydnophora microconos

Leptastrea solida

Montipora erythraea

Montipora granulata

Montipora tuberculosa

Plerogyra sinuosa

Podabacia crustacea

Pocillopora hemprichi

Porites solida

Porites somaliensis

Dendrophyllia micranthus

* Angaben nach Mergner \& Wedler (1977)

* Angaben nach Mergner (1979) 
die lebenden Reste einer einzigen, erheblich größeren Kolonie darstellen (z. B. Montipora stilosa in Einzelquadrat Ia, Favia stelligera in IId-IIId). Die geringe Größe dieser Einzelkolonien täuscht ein geringes Alter vor. Tatsächlich handelt es sich aber um alte Kolonien, deren Oberseiten größtenteils abgestorben, erodiert und von anderen Kolonien besiedelt sind. Hughes \& Jackson (1980) haben auf diese Problematik am Beispiel karibischer Korallen hingewiesen.

Die beiden Untersuchungen über den Cnidaria-Bestand des Testareals U-7 im Februar 1976 und im September 1977 erbrachten insgesamt 104 Arten: zu den in Tab. 1 aufgeführten 98 Arten kommen noch die drei Xenia-Arten: X. crassa, X. hicksoni und X. impulsatilla, die als winzige Einzelfunde nicht in die Karte, Abb. 3, eingetragen bzw. in Tab. 1 ausgewertet werden konnten, und die drei Aktinien Gyrostoma helianthus, G. quadricolor und Radianthus koseirensis, die zwar in der Karte, aber nicht in Tabelle 1 verzeichnet sind.

Im Gesamtbestand der determinierten Cnidaria-Arten fanden sich 78 ScleractiniaArten aus 34 Gattungen, 19 Alcyonaria-Arten aus 11 Gattungen, 3 Actiniaria-Arten aus 2 Gattungen und je eine Art der Hydroidea, Rhizostomeae, Stolonifera und Zoantharia. Eine derartig hohe Arten- und Gattungsdichte von Weich- und Steinkorallen wurde unseres Wissens noch in keinem Riffareal von nur $5 \times 5 \mathrm{~m}$ Fläche festgestellt. Für das Rote Meer bedeutet das etwa ein Drittel bis ein Viertel aller bisher bekannten Arten und 34 der 55 Gattungen.

Zusammen mit den schon früher (Mergner \& Schuhmacher, 1974; Mergner \& Wedler, 1977; Mergner, 1979) zusätzlich veröffentlichten Cnidarierfunden (Tab. 4) ergibt sich für die Riffe der jordanischen Küste des Golfes von Aqaba ein Bestand von 7 Hydroidenarten, 7 Aktinienarten, 101 Skleraktinienarten und 24 Alcyonarienarten, zusammen 139 Arten. Ihnen stehen von der Sinaiseite Listen mit u. a. 97 Skleraktinienarten (Loya \& Slobodkin, 1971) bzw. 99 Skleraktinienarten und 16 Oktokorallenarten (Benayahu \& Loya, 1977) gegenüber. Dazu kommen auch die von Schmidt (1972) veröffentlichten 18 Hydroidenarten.

Eine mehrtägig durchgeführte Kontrolle des Untersuchungsareales nach eineinhalb Jahren im September 1977 ergab gegenüber dem in den Abb. 2 und 3 dargestellten Zustand vom Februar 1976 eine Anzahl von Veränderungen im Besiedlungsbild der Cnidaria (Abb. 4). Neben der Neuansiedlung kleinster Jungkolonien, deren Anzahl auch nicht annäherungsweise festgestellt werden konnte (vgl. L-1; Mergner, 1979), waren inzwischen 27 Alcyonarienkolonien $(0,69 \%)$ und 53 Skleraktinienkolonien $(1,54$ $\%$ ) abgestorben mit zusammen $2,23 \%$ der Gesamtfläche der Cnidarierbedeckung und $1,11 \%$ des Gesamtareales (Tab. 3). Die eingetretenen Verluste betrafen unter den Weichkorallen vor allem die Xenia-Arten ( 23 Kolonien von 572) und unter den Steinkorallen Stylophora pistillata (4 Kolonien von 94), Montipora stilosa (4 von 61), Favia favus (4 von 25), Favites peresi (5 von 65) und Goniastrea pectinata (6 von 162), bei allen übrigen Arten nur jeweils 1-3 Kolonien. Im ungünstigsten Fall (Porites [Synarea] convexa) waren 3 von 4 Kolonien ( $75 \%$ ) abgestorben, bei Seriatopora angulata 1 von 5 $(20 \%)$, bei 4 weiteren Arten 12-15\% und sonst durchweg unter $10 \%$, meist unter $1-3 \%$.

Die große Zahl sessiler Cnidaria-Arten mit insgesamt 104 Arten, zusammen mit der Zahl ihrer 2070 Einzelkolonien, begründet eine hohe Diversität. Ihr Index $H$ (Pielou, 1969) beträgt auf der Basis der Koloniezahl berechnet 3,67; auf der Basis der jeweiligen. Siedlungsfläche 3,42 . 


\section{DISKUSSION}

Alle Beobachtungen und Meßergebnisse zur Einflußnahme der abiotischen Faktoren auf Zusammensetzung und Besiedlungsdichte des Korallenbestandes im Testareal U-7 weisen, miteinander kombiniert, auf ein ausgeglichenes "ökologisches Klima" im Bereich des mittleren Vorriffes hin: Hier unterliegen Wasserbewegung, Temperatur, Salinität, Sauerstoffgehalt und Beleuchtungsintensität nur geringen Schwankungen, und Extremwerte sind selten. Die hier gebotenen Lebensbedingungen sind daher für ein breites Spektrum ökologischer Ansprüche noch erträglich; für eine beträchtliche Zahl von Arten erscheinen sie sogar optimal. Hinsichtlich der Produktivität von Steinkorallen und ihrer Lichtansprüche hat Svoboda (1978) bei einigen der untersuchten Arten (Fungia, Acropora, Stylophora spp.) in dieser Tiefenstufe sogar die höchsten Werte im Vertikalvergleich gemessen.

Der Tiefenbereich des mittleren Vorriffes, in dem das Testareal U-7 liegt, ist die Ubergangszone zwischen Arten, die im Seichtwasser, d. h. am oberen Riffhang und auf dem Riffdach, dominieren, und solchen, die hauptsächlich erst in größerer Tiefe vorkommen. Zur ersten Gruppe offensichtlich photophiler Arten gehören in den Riffen bei Aqaba: Millepora exesa, Tubipora musica, Lithophyton arboreum, Xenia macrospiculata, Pocillopora damicornis, Stylophora pistillata, Acropora spp., Goniastrea retiformis und Platygyra lamellina. Geringere Lichtansprüche haben hingegen offensichtlich: Dendronephthya sp., Nephthea albida, Leptoseris sp., Pachyseris speciosa, Alveopora daedalea, Caulastrea tumida und Echinophyllia aspera, weshalb diese vermutlich sciaphilen Arten erst im tieferen Vorriff häufig sind. Eine bevorzugte Verbreitung im mittleren Vorriff scheinen zu haben: Parerythropodium fulvum, Sarcophyton ehrenbergi, Heteroxenia fuscescens, Xenia spp., Seriatopora spp., Montipora spp., Pavona spp., Goniopora spp., Favites peresi, Goniastrea pectinata, Leptastrea spp., Galaxea fascicularis und Lobophyllia corymbosa. Hingegen sind Porites-Kolonien vom Riffdach bis in das tiefere Vorriff $(50 \mathrm{~m})$ gleichmäßig vertreten. Für den größten Teil der festgestellten Arten reichen die Funddaten allerdings noch nicht aus, um eine mögliche Zuordnung zu einer bestimmten Tiefenstufe feststellen zu können.

Eine ähnliche Selektionswirkung wie von der Beleuchtungsintensität geht auch voll der Häufigkeit des Wasseraustausches pro Zeit- und Volumeneinheit aus: Vor allem die Hydrokoralle Millepora dichotoma, aber auch verschiedene Steinkorallen (Acropora spp. sowie massig wachsende Poritidae und Faviidae) sind mit ihren größten Kolonien in der Zone heftigster Wasserbewegung (Riffkante und oberer Riffhang) vertreten. Hier ist zwar einerseits die Zufuhr von $\mathrm{O}_{2}$ und planktischer Nahrung sowie der rasche Abtransport schädlicher Sedimente und $\mathrm{CO}_{2}$-Ansammlungen maximal gewährleistet, anderseits ist aber auch die Gefährdung durch die unmittelbar zerstörende Wirkung der Wasserkräfte am größten. Der mit zunehmender Tiefe deutliche Rückgang im Auftreten dieser Arten (insbesondere von Millepora dichotoma) weist darauf hin, daß sie als photophilrheophil zu charakterisieren sind. Im Gegensatz hierzu sind die Korallenarten Dendrophyllia micranthus und Balanophyllia spp. aus den Schattenhöhlen des oberen Riffhanges als sciaphil-rheophil zu bezeichnen.

An den Riffen von Aqaba lassen sich im allgemeinen drei Tiefenstufen unterscheiden, die nicht unbedingt mit der Einteilung des Vorriffes übereinstimmen, aber durch das gehäufte Vorkommen bestimmter Korallenarten charakterisiert werden (Einzelhei- 

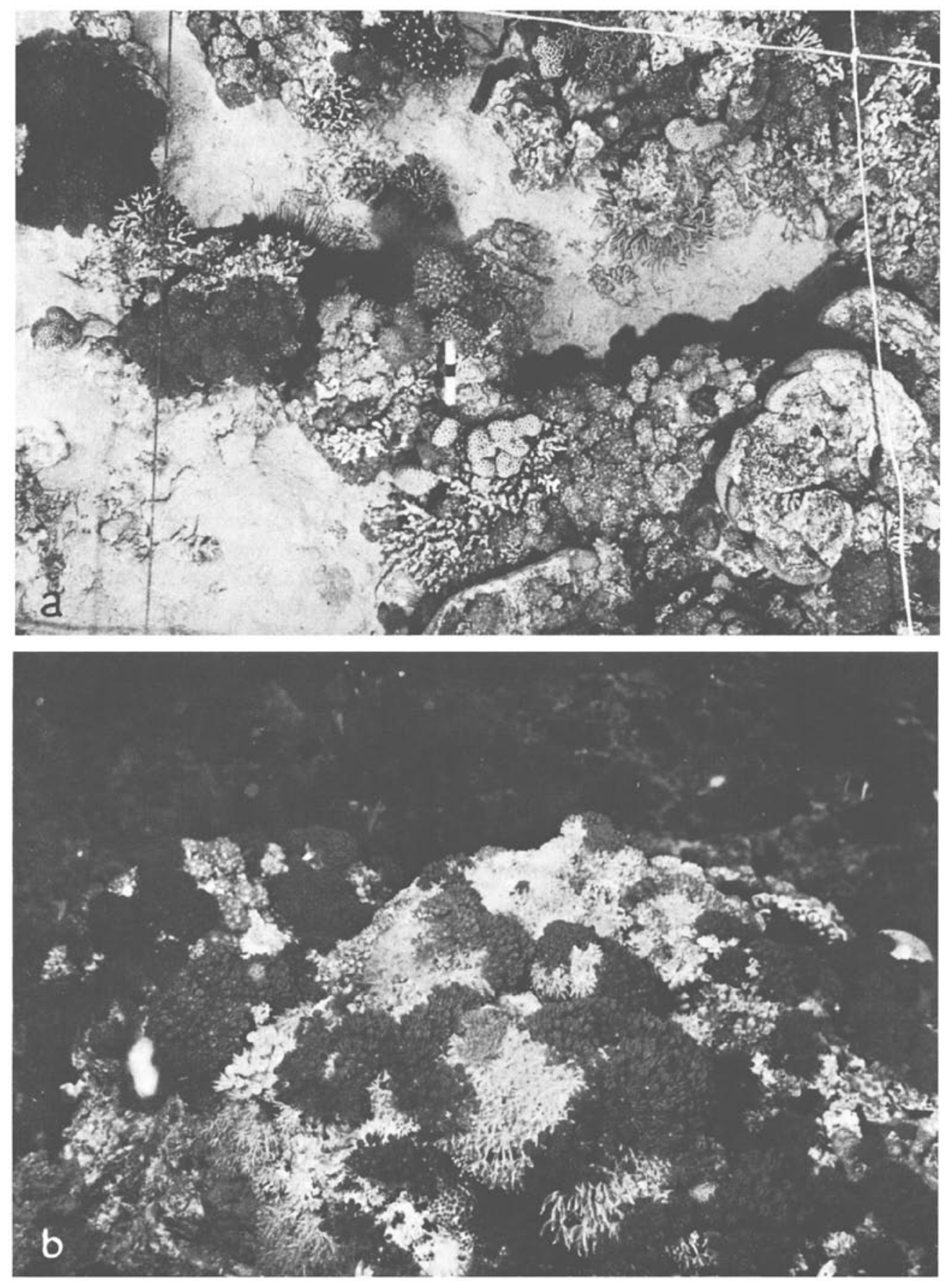

Abb. 5. Ausschnitte aus Testareal U-7. a. Unterwasserphotographie des Einzelquadrates IId. Aufnahmerichtung senkrecht von oben aus $2 \mathrm{~m}$ Höhe. b. Dichte Besiedlung einer großen abgestorbenen Steinkorallenkolonie durch verschiedene Xenia-Arten 
ten vgl. Mergner, 1980): Danach sind von den insgesamt 104 Cnidarierarten, die im Testareal U-7 festgestellt wurden, $50 \%$ in der obersten Tiefenstufe von $1-5 \mathrm{~m}$ häufig, $80 \%$ im mittleren Bereich von $5-15 \mathrm{~m}$ und ca. $40 \%$ in der unteren Tiefenstufe von 15-40 m. $10 \%$ haben eine ungefähr gleichmäßige Verbreitung über alle Tiefenstufen hinweg. Inwieweit die beobachteten Häufigkeitsverteilungen an den verschiedenen Standorten tatsächlich bevorzugten Lebensbedingungen der einzelnen Arten entsprechen oder aber zusätzlich das Resultat von (Raum-)Konkurrenzen widerspiegeln, können nur autökologisch-experimentelle Untersuchungen klarlegen.

Aus dem Charakter als Ubergangszone ohne ökologische Extrembedingungen erklärt es sich, daß das mittlere Vorriff einem breiten Spektrum ökologischer Ansprüche (noch) gerecht wird. So kommt es zu der enormen Artenfülle der Korallengemeinschaft in dieser Zone, die von keinem anderen Riffabschnitt bzw. Tiefenstufe erreicht wird.

Die geringe Koloniegröße bei den meisten im Testareal vertretenen Arten deutet freilich auf suboptimale Lebensbedingungen hin und läßt auf zwei ganz unterschiedliche Ursachen dafür schließen: (1) Schadwirkungen, die zugleich auch der Grund für die kahlen Korallenfelsflächen sind, und (2) interspezifische Konkurrenz um Siedlungsraum zwischen offenbar gleichwertigen Sedentarierarten.

Als lebensfeindlicher abiotischer Faktor kommt in dieser Tiefenregion nur die Sedimentation in Betracht. Wenngleich während des Untersuchungszeitraumes keine kritisch hohen Sedimentationsraten festzustellen waren, so muß aus dem Vorhandensein abgestorbener Scheitel bei vielen massigen Kolonien (so von Cyphastrea microphthalma, Favia spp., Favites peresi, Goniopora spp., Goniastrea pectinata u. a.) auf gelegentliche umfangreiche Sedimentablagerungen geschlossen werden. Sie können im nördlichen Golf von Aqaba vorkommen, wenn während der seltenen Südstürme ein mehrere Meter hoher Wellengang sich bis in diese Tiefen auswirkt und den Sand aus den Bodenmulden aufwirbelt.

Diademseeigel (Diadema setosum), die im Testquadrat häufig sind - die tagsüber versteckt sitzenden Tiere sind auf Abb. 2 z.B. in den Einzelquadraten Ia-c, IIb und IIIc gut zu erkennen -, erschweren durch ihre Weidetätigkeit die Wiederbesiedlung dieser kahlen Hartbodenflächen (zum Einfluß von Diadema setosum auf benachbarte Korallengemeinschaften vgl. auch Schuhmacher, 1974; Mergner, 1979; Mastaller, 1979; Mergner \& Mastaller, 1980). Eine weitere Ursache für das Vorkommen partiell abgestorbener Steinkorallenkolonien stellt die Raumkonkurrenz der üppig entwickelten Weichkorallenbestände dar, vor allem der Xeniiden, deren Anteil an der Cnidarierbedeckung mit über 600 Kolonien 35,81 \% ausmacht. Durch Abschatten und Behindern der Wasserzufuhr bringen sie die vergleichsweise kleinen Steinkorallenpolypen zum Absterben (Schuhmacher, 1975). Abb. 5b gibt einen guten Eindruck von einer derartigen, auf ehemaligen Korallenköpfen siedelnden Xenia-Ansammlung im Bereich des Testareals. Aus noch ungeklärten Gründen können die Xenia-Rasen aber auch binnen weniger Wochen wieder zurückgehen oder gänzlich verschwinden und hinterlassen dann freie Korallenfelsflächen für die sukzessive Wiederbesiedlung durch sessile Organismen.

Die aufgezeigten, ständigen Veränderungen im Besiedlungsbild, fủhren zugleich zu einer Erhöhung der Zahl der Einzelkolonien. Zusammen mit der zuvor begründeten eindrucksvollen Artendichte im Untersuchungsgebiet ergibt sich so der hohe Diversitätsindex von $H=3,67$ (bezogen auf die Koloniezahl) bzw. 3,42 (bezogen auf die Siedlungsflächen jeder Art). Er ist zugleich auch ein Hinweis dafür, daß die dicht 
zusammenlebenden und um Raum konkurrierenden Arten dennoch auf Dauer koexistieren können und die Inanspruchnahme der Siedlungsfläche durch eine oder wenige Arten hingegen unwahrscheinlich ist.

Die Befunde aus dem mittleren Vorriff zeigen, wie dies nicht anders zu erwarten war, wesentliche Unterschiede zu denen, die im gleichen Zeitraum (Früjahr 1976) im Testareal L-1 der Rifflagune erhoben wurden (Mergner \& Svoboda, 1977; Mergner, 1979; Mergner \& Mastaller, 1980). Während im mittleren Vorriff Cnidaria $42,36 \%$ der Gesamtarealfläche bedecken, sind es im Rifflagunenareal nur 6,60\%. Außerdem stehen den 104 Cnidarierarten des Vorriffareales mit ihren 2046 Einzelkolonien nur 18 Arten mit 354 Kolonien im Lagunenareal gegenüber und den Diversitätsindices $H=3,67$ bzw. $3,42 \mathrm{im}$ Vorriff ein solcher von $H=1,75$ in der Rifflagune. Im sessilen Benthos des mittleren Vorriffes dominieren eindeutig Cnidaria, im Bereich des Riffdaches dagegen Makroalgen mit 22 Arten und 2037 Kolonien bzw. Thalli, 26,07 \% Flächenbedeckung und einem Diversitätsindex $H=1$,14. Im Prinzip entsprechende Unterschiede zwischen Riffdach und Vorriff wurden von Benayahu \& Loya (1977) auf Grund von Transektzonierungen an verschiedenen Riffen der Sinaiküste des Golfes von Aqaba festgestellt.

Der Bewuchs der tiefer gelegenen Vorriffstufen wurde nur während gelegentlicher Tauchabstiege bis zu 50 m Tiefe abgeschätzt. Dabei sinkt die Rate lebender Bedeckung (im wesentlichen Cnidaria) deutlich ab. Hierfür ist neben den sich verschlechternden Lichtverhältnissen vor allem auch der steilere Neigungswinkel des Untergrundes verantwortlich. Von besonderem Interesse ist weiterhin ein Vergleich des untersuchten Vorriffabschnittes mit einem entsprechenden Abschnitt der gegenüberliegenden Sinaiküste des Golfes von Aqaba südlich Eilat. Dort hatten Loya \& Slobodkin (1971) und Loya (1972) die Korallenassoziationen verschiedener Tiefenzonen mit Hilfe der Transektmethode ermittelt und den mittleren Durchmesser verschiedener Korallenarten angegeben (Loya, 1972; Tab. 8). Unter der Annahme, daß dabei kreisförmige Projektionsflächen gemeint sind, ist ein Vergleich mit den Flächenangaben aus dem Testareal U-7 durchaus möglich. Dabei zeigt sich, daß die Kolonien der genannten Arten auf der östlichen Golfseite größer sind. Bei gleichem Bedeckungsanteil durch Cnidaria errechnet Loya (1972) allerdings einen niedrigeren Diversitätsindex, nämlich $H=2,83$. Doch sollte der Vergleich nicht zu weit getrieben werden, da bei der Testquadratmethode, wie sie bei Aqaba verwendet wurde, wesentlich mehr Kolonien ausgewertet werden als bei der von Loya angewandten Transektmethode. Immerhin zeichnet sich aber schon bei diesem Vergleich der Korallengemeinschaften aus etwa $10 \mathrm{~m}$ Tiefe $a b$, daß die ökologischen Bedingungen auf der Ostseite offensichtlich etwas besser sind. Diesen Befund hatten schon Mergner \& Schuhmacher (1974) auf Grund eines Vergleiches der ganzen Riffe erhoben.

Schließlich verdienen die hohe Artenzahl und Diversität auf engstem Raum auch unter dem Aspekt Beachtung, daß es sich bei dem untersuchten Standort um einen der nördlichsten Vorposten des Riffgürtels überhaupt handelt. Weltwelt ist nämlich ganz allgemein eine beträchtliche Abnahme der Anzahl der Korallenarten zum Rand des Riffgürtels hin zu beobachten (z. B.: Stehli \& Wells, 1971; Schuhmacher, 1976). Offensichtlich macht aber der nördliche Golf von Aqaba, dessen Korallen-Inventar nur unwesentlich geringer ist als das des mittleren Roten Meeres, eine Ausnahme von dieser Erscheinung. Der Grund für die nachweisliche Artenfülle des Golfes von Aqaba ist in seinen besonderen hydrographischen Bedingungen zu suchen (Mergner \& Schuhma- 
cher, 1974; Klinker et al., 1976). Wir erwarten in dieser Hinsicht von unseren Vergleichsuntersuchungen an Riffen der sudanesischen Küste weitere Aufschlüsse.

Danksagungen. Wir danken der Deutschen Forschungsgemeinschaft für die finanzielle Unterstützung unseres Aqaba-Projektes, der University of Jordan, Amman, für ihre Gastfreundschaft an der Marine Science Station Aqaba und den Herren Dr. M. Mastaller, F. Nobbe und Dr. A. Svoboda für ihre Hilfe bei der Anfertigung der Unterwasser-Photoserien bzw. bei der Aufsammlung von Belegstücken nicht unmittelbar ansprechbarer Korallenarten. Besonderer Dank gebührt auch den Kollegen, die taxonomisch schwierige Belegexemplare bestimmten: Dr. J. Versefeldt (Alcyonaria), Dr. G. Scheer und Dr. M. Wijsman-Best (Scleractinia). Schließlich dürfen wir Herrn H. Löseke für die rechnerischen Unterlagen der Tabellen und Herrn F. Theissen für die bewährte graphische Gestaltung unserer Abbildungsentwürfe danken. Herr Dr. M. Mastaller hat dankenswerterweise die Diversitätsindices berechnet. Der Farbdruck wurde aus Titel 52394, RUB, finanziert.

\section{ZITIERTE LITERATUR}

Benayahu, Y. \& Loya, Y., 1977. Space partitioning by stony corals, soft corals and benthic algae on the coral reefs of the northern Gulf of Eilat (Red Sea). - Helgoländer wiss. Meeresunters. 30 , $362-382$.

Bohnsack, J. A., 1979. Photographic quantitative sampling of hardbottom benthic communities. Bull, mar. Sci. 29, 242-252.

Dahl, A. L., 1973. Surface area in ecological analysis: Quantification of benthic coral reef algae.Mar. Biol. 23, 239-249.

Davies, S. P., Stoddart, D. R. \& Sigee, D. C., 1971. Reef forms of Addu Atoll, Maldives Islands. Symp. zool. Soc. Lond, 28, 217-259.

Dinesen, Z. D., 1977. The coral fauna of the Chagos Archipelago. - Proc. 3rd int. Coral Reef Symp. Miami 1, 155-161.

Goodwin, M. H., Cole, M. J. C., Stewart, W. E. \& Zimmerman, B. L., 1976. Species density and associations in Caribbean reef corals, - J. exp. mar. Biol. Ecol. 24, 19-31.

Hughes, T. P. \& Jackson, J. B. C., 1980. Do corals lie about their age? Some demographic consequences of partial mortality, fission, and fusion. - Science N. Y. 209, 713-715.

Klinker, J., Reiss, Z., Kropach, C., Levanon, I., Harpaz, H., Halicz, E. \& Assaf, G., 1976. Observations on the circulation pattern in the Gulf of Eilat (-Aqaba), Red Sea. - Israel J. Earth Sci. 25, 85-103.

Loya, Y., 1972. Community structure and species diversity of hermatypic corals at Eilat, Red Sea. Mar, Biol. 13, 100-123.

Loya, Y. \& Slobodkin, L. B., 1971. The coral reefs of Eilat (Gulf of Eilat, Red Sea). - Symp. zool. Soc. Lond. 28, 117-139.

Maragos, J. E., 1974. Coral communities on a seaward reef slope, Fanning Islands. - Pacif. Sci. 28, 257-278.

Mastaller, M., 1979. Beiträge zur Faunistik und Ökologie der Korallenriffe bei Aqaba, Rotes Meer, unter besonderer Berücksichtigung der Mollusken und Echinodermen. - Diss. Bochum, $337 \mathrm{pp}$.

Mergner, H., 1979. Quantitative ökologische Analyse eines Rifflagunenareals bei Aqaba (Golf von Aqaba, Rotes Meer). - Helgoländer wiss. Meeresunters. 32, 476-507.

Mergner, H., 1980. Ecology of a fore reef area near Aqaba (Red Sea).-Symp. coast. mar. Envir. Red Sea, Gulf of Aden and Tropical Western Indian Ocean, Karthoum (im Druck).

Mergner, H. \& Mastaller, M., 1980. Ecology of a reef lagoon near Aqaba (Red Sea). - Symp. coast. mar. Envir. Red Sea, Gulf of Aden and Tropical Western Indian Ocean, Karthoum (im Druck).

Mergner, H. \& Schuhmacher, H., 1974. Morphologie, Okologie und Zonierung von Korallenriffen bei Aqaba (Golf von Aqaba, Rotes Meer). - Helgoländer wiss. Meeresunters. 26, 238-358.

Mergner, H. \& Svoboda, A., 1977. Productivity and seasonal changes in selected reef areas in the Gulf of Aqaba (Red Sea). - Helgoländer wiss. Meeresunters. 30, 383-399.

Mergner, H. \& Wedler, E., 1977. Über die Hydroidpolypenfauna des Roten Meeres und seiner Ausgänge. - Meteor Forsch.-Ergebn. (D) 24, 1-32.

Morton, J., 1974. The coral reefs of the British Salomon Islands; a comparative study of their composition and ecology. - Proc. 2nd int. Coral Reef Symp. Brisbane 2, 31-54. 
Neudecker, S., 1976. Effects of thermal effluent on the coral reef community at Tanguisson. - Univ. Guam Mar, Lab. Techn. Rep. 30, 1-55.

Ott, B. \& Auclair, A. N., 1977. Cluster analytic definition of species ecological groups for a submerged barrier reef in Barbados, West Indies. - Int. Revue ges. Hydrobiol. 62, 41-51.

Pichon, M., 1978. Problems of measuring and mapping coral reef colonies. In: Coral reefs: Research methods. Ed. by D. R. Stoddart \& R. E. Johannes. Unesco, Paris 219-230. (Monographs on oceanographic methodology. 5).

Pielou, E. C., 1969. An introduction to mathematical ecology. New York, Wiley-Interscience, $286 \mathrm{pp}$.

Salvat, B., 1972. La faune benthique du lagon de l'atoll de Reao (Tuamotu, Pol. franç.). - Cah. Pacif. $16,31-110$.

Schmidt, H. E., 1972. Some new records of hydroids from the Gulf of Aqaba with zoogeographical remarks on the Red Sea area. - J. mar. biol. Ass. India 13, $27-51$.

Schuhmacher, H., 1974. On the conditions accompanying the first settlement of corals on artificial reefs with special reference to the influence of grazing sea urchins (Eilat, Red Sea). - Proc. 2nd int. Coral Reef Symp. Brisbane 1, 257-267.

Schuhmacher, H., 1975. Die Rolle der Weichkorallen (Alcyonacea, Octocorallia) innerhalb der Riffbiozönosen des Roten Meeres und des australischen Großen Barriereriffes. - Verh. dt. zool. Ges. 67, 380-384.

Schuhmacher, H., 1976. Korallenriffe. - BLV, München, $275 \mathrm{pp}$.

Stehli, F. G. \& Wells, J. W., 1971. Diversity and age patterns in hermatypic corals. - Syst. Zool. 20 , $115-127$.

Svoboda, A., 1978. In situ monitoring of oxygen production and respiration in Cnidaria with and without zooxanthellae. In: Physiology and behaviour of marine organisms. Ed. by D. S. McLusky \& A. J. Berry. Pergamon Press, Oxford, 75-82. 